\title{
A Block Cipher Mode of Operation with Two Keys
}

\author{
Yi-Li Huang, Fang-Yie Leu, Jung-Chun Liu, and Jing-Hao Yang \\ Department of Computer Science, TungHai University, Taiwan \\ \{yifung, leufy, jcliu,g01350036\}@thu.edu.tw
}

\begin{abstract}
In this paper, we propose a novel block cipher mode of operation (BCMO for short), named Output Protection Chain (OPC for short), which as a symmetric encryption structure is different from other existing BCMOs in that it employs two keys, rather than one key, to protect the output of the mode. The security threats of chosen-plaintext attacks on three existing common BCMOs, including the Cipher Feedback mode (CFB), the Output Feedback mode (OFB), and the Counter mode (CTR), are also analyzed. After that, we explain why the OPC mode (or simply the OPC) can effectively avoid chosen-plaintext attacks, and why its security level is higher than those of CFB, OFB, and CTR.
\end{abstract}

Keywords: Block cipher, Cipher Feedback mode, Output Feedback mode, Counter mode, Output Protection Chain mode, chosen-plaintext attack.

\section{Introduction}

When standard block cipher algorithms, like Data Encryption Standard (DES), Triple Data Encryption Algorithm (3DES), and Advanced Encryption Standard (AES), are used to encrypt a plaintext block, the size of the block should be the same as the length of the encrypting key (or called the ciphering block) L. If the size exceeds L, we have to divide the plaintext block into sub-blocks. Each is L in length. Several BCMOs defined by National Institute of Standards and Technology (NIST) have been widely adopted by different block cipher techniques [2]. Through the use of these BCMOs, these techniques can be then applied to many applications.

Generally, the standard BCMOs include the cipher Feedback mode (CFB for short), the Output Feedback mode (OFB for short) and the Counter mode (CTR for short), the characteristics of which are that they use only one key to encrypt multiple plaintext blocks, and the efficiencies of their block cipher algorithms are high [1]. Currently, different types of attacks on these BCMOs have been developed [3] [4], meaning the BCMOs have their own security problems. Therefore, in this study, we propose a novel BCMO, named Output Protection Chain (OPC for short), to solve the existing BCMOs' security problems. Two different structures of the OPC, named OPC-1 and OPC-2, have been developed to enhance the security levels of BCMOs. We will describe the two structures later.

\section{Block Cipher Modes of Operation}

Before describing operations of the CFB, OFB, and CTR, we first define the parameters used by them. 
$\mathrm{P}_{\mathrm{i}}$ : The $\mathrm{i}^{\text {th }}$ plaintext block to be encrypted, $1 \leq \mathrm{i} \leq \mathrm{n}$.

$\mathrm{C}_{\mathrm{i}}$ : The $\mathrm{i}^{\text {th }}$ ciphertext block, $1 \leq \mathrm{i} \leq \mathrm{n}$.

Block Cipher Encryption (BCE) unit: According to [2], the standard BCE units are AES-128, AES-192, and AES-256. The function of a BCE unit is denoted by $\mathrm{E}\left(\mathrm{I}_{\mathrm{P}}\right.$, $\mathrm{K}$ ), in which the key $\mathrm{K}$ and the input $\mathrm{I}_{\mathrm{p}}$ are used to encrypt a given plaintext block.

$\mathrm{K}$ : The block cipher key [2].

$\mathrm{O}_{\mathrm{i}}$ : The output block produced by invoking the $\mathrm{E}\left(\mathrm{I}_{\mathrm{p}}, \mathrm{K}\right), 1 \leq \mathrm{i} \leq \mathrm{n}$.

cr: The counter, which is an input of the BCE unit of the CTR.

IV: Initialization Vector (IV for short), a random value employed by the CFB and OFB since they need an additional initial input.

The general rule in $\mathrm{CFB}$ is that $\mathrm{E}\left(\mathrm{C}_{\mathrm{i}-1}, \mathrm{~K}\right)$ receives $\mathrm{C}_{\mathrm{i}-1}$ and $\mathrm{K}$ as its inputs to generate $\mathrm{O}_{\mathrm{i}}$ which is then XORed with $\mathrm{P}_{\mathrm{i}}$ to produce $\mathrm{C}_{\mathrm{i}}, 1 \leq \mathrm{i} \leq \mathrm{n}$, where $\mathrm{C}_{0}=\mathrm{IV}$. The process can be formulated as follows.

$$
\mathrm{C}_{\mathrm{i}}=\mathrm{P}_{\mathrm{i}} \oplus \mathrm{E}\left(\mathrm{C}_{\mathrm{i}-1}, \mathrm{~K}\right)=\mathrm{P}_{\mathrm{i}} \oplus \mathrm{O}_{\mathrm{i}}
$$

The encryption operations of the OFB are similar to those of the CFB. The difference is the inputs of $\mathrm{E}\left(\mathrm{I}_{\mathrm{P}}, \mathrm{K}\right)$. In the $\mathrm{OFB}, \mathrm{O}_{\mathrm{i}-1}$, rather than $\mathrm{C}_{\mathrm{i}-1}$, is fed back to the BCE unit to generate $\mathrm{O}_{\mathrm{i}}, 1 \leq \mathrm{i} \leq \mathrm{n}$, where $\mathrm{O}_{0}=$ IV. It can be formulated as follows.

$$
\mathrm{C}_{\mathrm{i}}=\mathrm{P}_{\mathrm{i}} \oplus \mathrm{E}\left(\mathrm{O}_{\mathrm{i}-1}, \mathrm{~K}\right)=\mathrm{P}_{\mathrm{i}} \oplus \mathrm{O}_{\mathrm{i}}
$$

The CTR encryption replaces the feedback operation employed by the CFB and OFB with a counter $\mathrm{cr}$ as one of the inputs of the BCE unit to generate $\mathrm{O}_{\mathrm{i}}$. The value of the counter used to generate $\mathrm{O}_{\mathrm{i}}$ is $\mathrm{cr}+\mathrm{i}-1$ where $\mathrm{cr}$ is the value adopted to produce $\mathrm{O}_{\mathrm{i}}, 1 \leq \mathrm{i} \leq \mathrm{n}$. The formulas utilized to encrypt plaintext blocks of the CTR are as follows.

$$
\mathrm{C}_{\mathrm{i}}=\mathrm{P}_{\mathrm{i}} \oplus \mathrm{E}(\mathrm{cr}+\mathrm{i}-1, \mathrm{~K})
$$

\section{The Output Protection Chain (The OPC)}

In this section, we describe how to encrypt plaintext blocks and decrypt ciphertext blocks in the proposed OPC structures, i.e., OPC-1 and OPC-2. We first define those parameters and functions invoked by the OPCs.

The definitions of $\mathrm{P}_{\mathrm{i}}, \mathrm{C}_{\mathrm{i}}, \mathrm{BCE}$ units, $\mathrm{E}\left(\mathrm{I}_{\mathrm{p}}, \mathrm{K}\right)$ and $\mathrm{O}_{\mathrm{i}}, 1 \leq \mathrm{i} \leq \mathrm{n}$, are the same as those defined above. New parameters, operations and functions are defined below.

Key1: The block cipher key, the role of which is the same as $\mathrm{K}$ defined above.

$\mathrm{D}\left(\mathrm{I}_{\mathrm{p}}, \mathrm{K}\right)$ : Function of the block decipher, in which the key $\mathrm{K}$ and an input $\mathrm{I}_{\mathrm{P}}$ are used to decrypt a plaintext block from its ciphertext block.

$\mathrm{G}_{\mathrm{i}}$ : The data block produced by $\mathrm{O}_{\mathrm{i}} \oplus \mathrm{P}_{\mathrm{i}}, 1 \leq \mathrm{i} \leq \mathrm{n}$.

Key2: A key with the length the same as that of $\mathrm{O}_{\mathrm{i}}$. It is used to encrypt $\mathrm{G}_{1}$ in the

OPC-1, and $\mathrm{O}_{1}$ in the OPC-2.

$+_{2}$ : a binary adder, which is a logical operator defined in [5].

$-_{2}$ : The Inverse operation of $+_{2}$. 


\subsection{The OPC-1}

As shown in Fig.1, the general rule of the OPC-1 is that Key1 and $G_{i-1}$ are input to the BCE unit to generate $O_{i}$, which is XORed with $P_{i}$ to produce $G_{i} \cdot G_{i}$ is then binaryadded with $\mathrm{O}_{\mathrm{i}-1}$ to generate $\mathrm{C}_{\mathrm{i}}, 1 \leq \mathrm{i} \leq \mathrm{n}$. The formulas derived are as follows.

$$
\mathrm{C}_{\mathrm{i}}=\left[\mathrm{E}\left(\mathrm{G}_{\mathrm{i}-1}, \mathrm{Key} 1\right) \oplus \mathrm{P}_{\mathrm{i}}\right]+{ }_{2} \mathrm{O}_{\mathrm{i}-1}=\mathrm{G}_{\mathrm{i}}+{ }_{2} \mathrm{O}_{\mathrm{i}-1}
$$

where $\mathrm{G}_{0}=\mathrm{IV}$ and $\mathrm{O}_{0}=$ Key2. The decryption process as shown in Fig. 2 can be formulated as follows.

$$
\mathrm{P}_{\mathrm{i}}=\mathrm{O}_{\mathrm{i}} \oplus \mathrm{G}_{\mathrm{i}}=\mathrm{E}\left(\mathrm{G}_{\mathrm{i}-1}, \mathrm{Key} 1\right) \oplus\left(\mathrm{C}_{\mathrm{i}}-{ }_{2} \mathrm{O}_{\mathrm{i}-1}\right)
$$

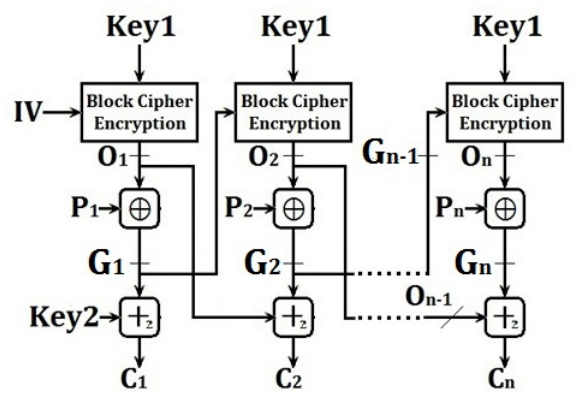

Fig. 1. The OPC-1 encryption

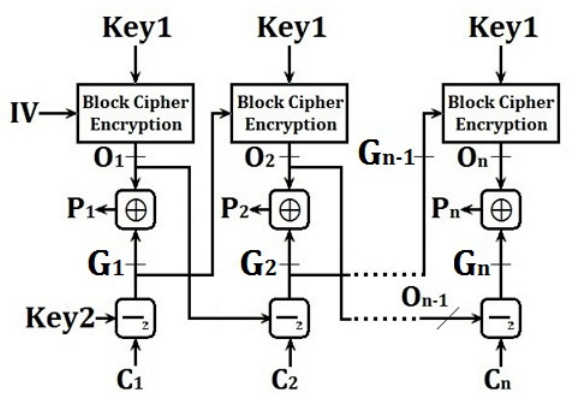

Fig. 2. The OPC-1 decryption

\subsection{The OPC-2}

The encryption process of the OPC-2 is shown in Fig. 3. The general rule is that $P_{i}$ and Key1 are input to the BCE unit to generate $\mathrm{O}_{\mathrm{i}}$, which is XORed with $\mathrm{O}_{\mathrm{i}-1}$ to generate $\mathrm{C}_{\mathrm{i}}, 1 \leq \mathrm{i} \leq \mathrm{n}$. It can be formulated as follows.

$$
\mathrm{C}_{\mathrm{i}}=\mathrm{O}_{\mathrm{i}} \oplus \mathrm{O}_{\mathrm{i}-1}=\mathrm{E}\left(\mathrm{P}_{\mathrm{i}}, \mathrm{Key} 1\right) \oplus \mathrm{O}_{\mathrm{i}-1}
$$

where $\mathrm{O}_{0}=$ Key2. The decryption structure of the OPC-2 as shown in Fig. 4 is as follows. To decrypt $C_{i}$, one needs $\mathrm{O}_{\mathrm{i}-1}$ to calculate $\mathrm{O}_{\mathrm{i}}$ because $\mathrm{O}_{\mathrm{i}}=\mathrm{C}_{\mathrm{i}} \oplus \mathrm{O}_{\mathrm{i}-1}$, $1 \leq \mathrm{i} \leq \mathrm{n}$. $\mathrm{P}_{\mathrm{i}}$ can be obtained by invoking the following formulas.

$$
\mathrm{P}_{\mathrm{i}}=\mathrm{D}\left(\mathrm{C}_{\mathrm{i}} \oplus \mathrm{O}_{\mathrm{i}-1}, \text { Key1 }\right)=\mathrm{D}\left(\mathrm{O}_{\mathrm{i}}, \text { Key1 }\right)
$$

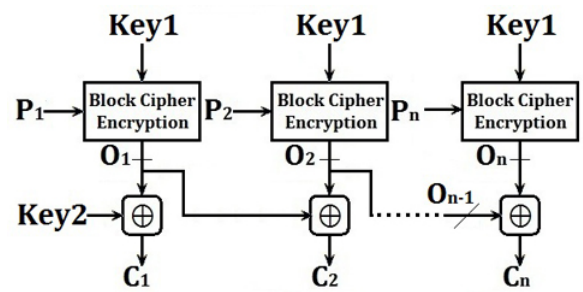

Fig. 3. The OPC-2 encryption

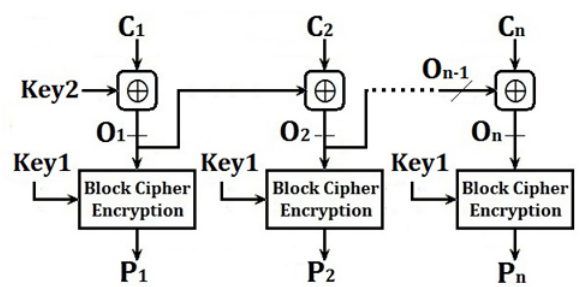

Fig. 4. The OPC-2 decryption 


\section{Security Analysis}

The advantage of using BCMOs is that these BCMOs can enhance security of a single block's encryption. Even if the block cipher (e.g. DES) has been cracked, in order to improve the security level of a security system, one can apply the DES as the BCE unit to the BCMOs. We will analyze the security of BCMOs mentioned above in the following subsections.

\subsection{Security of the CFB}

To launch a chosen-plaintext attack, an attacker first inputs $\mathrm{n}$ different plaintext blocks, denoted by $\mathrm{P}=\left\{\mathrm{P}_{1}, \mathrm{P}_{2}, \ldots, \mathrm{P}_{\mathrm{n}}\right\}$, to acquire a set of $\mathrm{n}$ ciphertext blocks, denoted by $\mathrm{C}=\left\{\mathrm{C}_{1}, \mathrm{C}_{2}, \ldots, \mathrm{C}_{\mathrm{n}}\right\}$, where $\mathrm{P}_{\mathrm{i}}$ is the $\mathrm{i}^{\text {th }}$ block of $\mathrm{P}$, and $\mathrm{C}_{\mathrm{i}}$ is the $\mathrm{i}^{\text {th }}$ block of $\mathrm{C}$, $1 \leq \mathrm{i} \leq \mathrm{n}$. In the CFB, $\mathrm{O}_{\mathrm{i}}$ can be derived from $\mathrm{P}_{\mathrm{i}}$ and $\mathrm{C}_{\mathrm{i}}$ since $\mathrm{O}_{\mathrm{i}}=\mathrm{P}_{\mathrm{i}} \oplus \mathrm{C}_{\mathrm{i}}$. If $\mathrm{n}$ is huge, the attacker can then collect sufficient $\left\langle\mathrm{C}_{\mathrm{i}-1}, \mathrm{O}_{\mathrm{i}}\right\rangle$ pairs, as the input and output of the BCE unit when encrypting $\mathrm{P}_{\mathrm{i}}$, to analyze the value of the key $\mathrm{K}$.

\subsection{Security of the OFB}

For the OFB, we analyze its security based on two cases, one is that the IV can be chosen by users, and the other is cannot be chosen.

\subsubsection{Attack on IV Able to be Chosen}

In the OFB, $\mathrm{O}_{\mathrm{i}}, 1 \leq \mathrm{i} \leq \mathrm{n}$, is only determined by IV and $\mathrm{K}$. If IV can be chosen by users, the attacker can select the IV the same as the one chosen by a user, i.e., the victim, to encrypt their chosen-plaintext and calculate $O_{i}$ by using $P_{i} \oplus C_{i}$.

Since $\mathrm{K}$ and the encryption algorithm of the BCE unit when encrypting different plaintext blocks are themselves the same, that means once the chosen IVs for encrypting two plaintexts are the same. When $\mathrm{O}_{\mathrm{i}} \mathrm{s}$ of the $\mathrm{BCE}$ unit are acquired, the attacker can use an illegally intercepted $\mathrm{C}_{\mathrm{i}}$ to search the corresponding $\mathrm{O}_{\mathrm{i}}$ from all its collected $\left\langle\mathrm{C}_{\mathrm{i}}, \mathrm{O}_{\mathrm{i}}\right\rangle$ pairs to derive $\mathrm{P}_{\mathrm{i}}$ without requiring breaking the key $\mathrm{K}$ of the $\mathrm{BCE}$ unit, since $\mathrm{P}_{\mathrm{i}}=\mathrm{C}_{\mathrm{i}} \oplus \mathrm{O}_{\mathrm{i}}$.

\subsubsection{Attack on IV Unable to be Chosen}

If the IV cannot be chosen, the security level of the OFB is higher. But it still faces the same security problem of the CFB. Like that in attacking the CFB, the attacker can first input a long plaintext, $\mathrm{P}=\left\{\mathrm{P}_{1}, \mathrm{P}_{2}, \ldots, \mathrm{P}_{\mathrm{n}}\right\}$, to acquire the corresponding ciphertext, $C=\left\{C_{1}, C_{2}, \ldots, C_{n}\right\}$, so as to generate a set of $O=\left\{O_{1}, O_{2}, \ldots, O_{n}\right\}$ since $\mathrm{O}_{\mathrm{i}}=\mathrm{P}_{\mathrm{i}} \oplus \mathrm{C}_{\mathrm{i}}$.

If $n$ is huge enough, the attacker can then collect sufficient $\left\langle\mathrm{O}_{\mathrm{i}-1}, \mathrm{O}_{\mathrm{i}}\right\rangle$ pairs to analyze the key K of its BCE unit. After that, when the attacker eavesdrops the messages delivered between the sender and receiver, and retrieves the IV, he/she can generate $\mathrm{O}$ to decrypt the intercepted $\mathrm{C}$ so as to obtain $\mathrm{P}$ since $\mathrm{P}=\mathrm{C} \oplus \mathrm{O}$. 


\subsection{Security of the CTR}

Like that in the OFB, the CTR can also be divided into two cases, i.e., a user can and cannot choose the value of $\mathrm{cr}$.

\subsubsection{Attack on CR Able to be Chosen}

In the CTR encryption, $\mathrm{O}_{\mathrm{i}} \mathrm{s}$ are determined only by $\mathrm{cr}$ and $\mathrm{K}$ in which $\mathrm{cr}$ is an incremental integer (will be transformed into a bit string) and $\mathrm{K}$ is a fixed key. The general rule is that given a chosen $\mathrm{cr}, \mathrm{E}(\mathrm{cr}+\mathrm{i}-1, \mathrm{~K})$ receives $\mathrm{cr}+\mathrm{i}-1$ and $\mathrm{K}$, $1 \leq \mathrm{i} \leq \mathrm{n}$, as its inputs to generate a set of output $\mathrm{O}=\left\{\mathrm{O}_{1}, \mathrm{O}_{2}, \ldots, \mathrm{O}_{\mathrm{n}}\right\}, \mathrm{n} \gg 1$, without requiring inputting any plaintext. On the other hand, for the chosen cr, the attacker can choose a plaintext $\mathrm{P}=\left\{\mathrm{P}_{1}, \mathrm{P}_{2}, \ldots, \mathrm{P}_{\mathrm{n}}\right\}$ for the CTR to generate a ciphertext $\mathrm{C}=\left\{\mathrm{C}_{1}, \mathrm{C}_{2}, \ldots, \mathrm{C}_{\mathrm{n}}\right\}$. After that, for each $\mathrm{i}, 1 \leq \mathrm{i} \leq \mathrm{n}, \mathrm{O}_{\mathrm{i}}=\mathrm{C}_{\mathrm{i}} \oplus \mathrm{P}_{\mathrm{i}}$. Then the attacker can acquire $\mathrm{O}$.

If users can choose cr, then the attacker can also choose a cr the same as that of a user, i.e., the victim, to obtain $\mathrm{O}$ corresponding to this cr by using the above process. Now the attacker can decrypt the plaintext block $P_{i}$ from the ciphertext block $C_{i}$ intercepted from the user by using $\mathrm{P}_{\mathrm{i}}=\mathrm{C}_{\mathrm{i}} \oplus \mathrm{O}_{\mathrm{i}}$.

\subsubsection{Attack on CR Unable to be Chosen}

If the cr cannot be chosen, the attacker is still able to know the cr. Because cr is delivered together with $\mathrm{C}_{\mathrm{i}} \mathrm{s}$ to the receiver with cr unencrypted [6], the attacker can obtain $\mathrm{n}$ input blocks, i.e., $\mathrm{I}_{\mathrm{p}}=\{\mathrm{cr}, \mathrm{cr}+1, \ldots, \mathrm{cr}+\mathrm{i}-1\}, \mathrm{n} \gg 1$, from those messages carrying crs and $\mathrm{C}_{\mathrm{i}} \mathrm{s}$.

On the other hand, the attacker can input a set of plaintext blocks $\mathrm{P}=\left\{\mathrm{P}_{1}, \mathrm{P}_{2}, \ldots, \mathrm{P}_{\mathrm{n}}\right\}$ to the CTR to obtain the corresponding ciphertext blocks, denoted by $\mathrm{C}=\left\{\mathrm{C}_{1}, \mathrm{C}_{2}, \ldots, \mathrm{C}_{\mathrm{n}}\right\}$. He/she can then acquire a set of outputs of the BCE unit, denoted by $\mathrm{O}=\left\{\mathrm{O}_{1}, \mathrm{O}_{2}, \ldots, \mathrm{O}_{\mathrm{n}}\right\}$, since $\mathrm{O}_{\mathrm{i}}=\mathrm{P}_{\mathrm{i}} \oplus \mathrm{C}_{\mathrm{i}}, 1 \leq \mathrm{i} \leq \mathrm{n}$. As a result, the attacker can analyze the key $\mathrm{K}$ used by the $\mathrm{BCE}$ unit after collecting a large number of $\left\langle\mathrm{cr}+\mathrm{i}-1, \mathrm{O}_{\mathrm{i}}\right\rangle$ pairs.

\subsection{Security of the OPC-1}

In Fig. 1, we use Key2 to protect $G_{1}$ and produce $C_{1}$, where $G_{1}=P_{1} \oplus O_{1}$. After that, $\mathrm{O}_{\mathrm{i}}$, $\mathrm{i}>1$, as the new Key2 of the next encryption round, is used to encrypt $\mathrm{G}_{\mathrm{i}+1}$ to generate $\mathrm{C}_{\mathrm{i}+1}$. The advantage is that, when a large number of chosenplaintext is input, $\mathrm{C}_{\mathrm{i}}$ collected by the attacker is the one encrypted by $\mathrm{O}_{\mathrm{i}-1}$ or Key2 (when $\mathrm{i}=1$ ). So there is no way for the attacker to decrypt $\mathrm{G}_{\mathrm{i}} \mathrm{s}$ by using an inverse operation on $\mathrm{C}_{\mathrm{i}} \mathrm{s}$ without knowing Key2 beforehand.

Moreover, $G_{i}$ is fed back to generate $\mathrm{O}_{i+1}$. The purpose is to increase the complexity of solving Key1. Also, $\mathrm{G}_{\mathrm{i}}$ is encrypted by $\mathrm{O}_{\mathrm{i}-1}$ or Key2, resulting in the fact that it is hard for the attacker to analyze the relationship between $P_{i}$ and $C_{i}, 1 \leq i \leq n$.

The shortcoming of the OPC-1 is that the plaintext is not encrypted by the BCE unit so the possibility for the attacker to decrypt $P_{i}$ from $C_{i}$ is still high since the attacker does not need to decrypt the BCE unit. 


\subsection{Security of the OPC-2}

Fig. 3 shows the OPC-2 encryption, in which Key2 is used to encrypt $O_{1}$ so as to produce $C_{1}$. Meanwhile, $\mathrm{O}_{1}$ is also used to encrypt $\mathrm{O}_{2}$, i.e., $\mathrm{C}_{\mathrm{i}}=\mathrm{O}_{\mathrm{i}} \oplus \mathrm{O}_{\mathrm{i}-1}$, $1<i \leq n$. As with the OPC-1, it is hard for the attacker to acquire $\mathrm{O}_{\mathrm{i}}$ by decrypting $\mathrm{C}_{\mathrm{i}}$ since $\mathrm{O}_{\mathrm{i}}$ is encrypted by $\mathrm{O}_{\mathrm{i}-1}$ or Key2, even he/she has collected a large number of ciphertext by inputting many chosen-plaintexts to the OPC-2.

If the attacker wishes to analyze the BCE unit of the OPC-2, he/she can input a long plaintext $\mathrm{P}=\left\{\mathrm{P}_{1}, \mathrm{P}_{2}, \ldots, \mathrm{P}_{\mathrm{n}}\right\}$ of $\mathrm{n}$ plaintext blocks to the OPC-2 to generate the corresponding ciphertext $\mathrm{C}=\left\{\mathrm{C}_{1}, \mathrm{C}_{2}, \ldots, \mathrm{C}_{\mathrm{n}}\right\}$. But before generating the set of output $\mathrm{O}=\left\{\mathrm{O}_{1}, \mathrm{O}_{2}, \ldots, \mathrm{O}_{\mathrm{n}}\right\}$ of the $\mathrm{BCE}$ unit, he/she still needs to know Key2 because $\mathrm{O}_{1}=\mathrm{C}_{1} \oplus$ Key2 , and $\mathrm{O}_{\mathrm{i}}=\mathrm{C}_{\mathrm{i}} \oplus \mathrm{O}_{\mathrm{i}-1}, 1<i \leq n$. In our design, all encryption and decryption steps are dependent, so it is impossible to acquire $\mathrm{O}_{1}$ without knowing Key2. Moreover, $\mathrm{P}_{\mathrm{i}}$ is also protected by the $\mathrm{BCE}$ unit. With this, OPC-2 effectively strengthens the security level of $\mathrm{O}_{\mathrm{i}}$. As a result, it is hard for the attacker to collect sufficient information to analyze Keyl of the BCE unit.

\section{Conclusions and Future Studies}

In this paper, we describe the security drawbacks of the standard BCMOs, and propose the OPCs to improve the security level of a block ciphering system by protecting the outputs of its $\mathrm{BCE}$ unit, i.e., $\mathrm{O}_{\mathrm{i}} \mathrm{s}$, without the need of preventing the attacker from collecting $\mathrm{P}_{\mathrm{i}} \mathrm{s}, \mathrm{C}_{\mathrm{i}} \mathrm{s}$ and their relationship. The purpose is avoiding the security system from being attacked by known or chosen-plaintext/ciphertext attacks.

However, in the OPC-2, the BCE unit must be invertible, e.g., DES, 3-DES, or AES. Otherwise, the plaintext $P_{i}$ cannot be reverted from $O_{i}$. Since the encryption speeds of non-invertible algorithms are often short, and their encryption keys are difficult to crack, if one replaces the BCE unit of the CFB, OFB, CTR or OPC-1 with a non-invertible algorithm, the security levels and the processing performance of these BCMOs will be then higher than before. Therefore, in the future, we will apply noninvertible algorithms to the OPC-1 so as to propose a new BCMO with the security at least the same as or higher than those of the two OPCs.

Acknowledgements. This research was partially supported by TungHai University on GREENs project, and National Science Council, Taiwan, grants NSC 100-2221-E029-018 and 101-2221-E-029-003-MY3.

\section{References}

1. Stallings, W.: Cryptography and Network Security: Principles and Practice, 5th edn. Prentice Hall (January 2010)

2. National Institute of Standards and Technology, NIST Special Publication 800-38A, Recommendation for Block Cipher Modes of Operation Methods and Techniques (December 2001) 
3. Hudde, H.: Building Stream Ciphers from Block Ciphers and their Security. Seminararbeit Ruhr-Universität Bochum (February 2009),

http://imperia.rz.rub.de:9085/imperia/md/content/

seminare/itsws08_09/hudde.pdf

4. Wang, D., Lin, D., Wu, W.: Related-Mode Attacks on CTR Encryption Mode. International Journal of Network Security 4(3), 282-287 (2007)

5. Huang, Y.F., Leu, F.Y., Chiu, C.H., Lin, I.L.: Improving Security Levels of IEEE802.16e Authentication by Involving Diffie-Hellman PKDS. Journal of Universal Computer Science 17(6), 891-911 (2011)

6. Lipmaa, H., Rogaway, P., Wagner, D.: Comments to NIST concerning AES Modes of Operations: CTR-Mode Encryption (2000), http: / / csrc.nist.gov/ 